\title{
COUPLING CORRECTION OF A CIRCULARLY POLARIZING UNDULATOR AT THE ADVANCED PHOTON SOURCE*
}

\author{
L. Emery ${ }^{\dagger}$ \\ Argonne National Laboratory, Argonne, IL 60439, USA
}

\begin{abstract}
The electromagnetic circularly polarizing undulator (CPU) installed at the Advanced Photon Source (APS) storage ring produces skew quadrupole field errors, which were initially corrected by a small skew quadrupole magnet at one end of the device. Because the storage ring is operated at $1 \%$ coupling or less, a correction not located at the source inside the CPU is insufficient, as we have confirmed in simulation. Adding a skew coil at the other end of the CPU allows us to make a complete correction of the coupling source in the undulator. Correction set points are determined by APS's general optimizing software with the vertical beam size of an $\mathrm{x}$-ray pinhole image as a readback.
\end{abstract}

\section{INTRODUCTION}

The circularly polarizing undulator (CPU) installed in ID4 of the Advanced Photon Source storage ring produces magnetic field errors, which can be reduced via application of corrective fields by the seven small dipole and mulitpole magnets located at the ends of the device.

Because the errors depend on the polarization mode and the main coil set points, a feedforward system had been designed to automatically send predetermined settings to the seven magnets according to the basic operating variables. In particular, a single skew quadrupole magnet was devised to compensate the integrated skew quadrupole field error of the CPU.

In general, a feedforward table for each polarization mode is determined from measurements at each main coil set point involving a scan or an optimization of the correction coil set points. Since this procedure disturbs the stored beam, this process has to be done during machine studies. Once the optimum set points of the coils are known for a set of main coil set points, the data are collected into a table that is loaded into the control system of the CPU. During user operations, the correction magnet set points are continuously updated by a table interpolator in the control system.

To correct the skew quadrupole field error we use the measurement of the vertical beam size, which is a substitute quantity for beam coupling and beam $x-y$ coupling. (The beam is imaged at a point where $\beta_{y}=27 \mathrm{~m}$ using an X-ray pinhole camera of resolution $22 \mu \mathrm{m}$.) The vertical beam size is first minimized using the ring's nineteen skew quadrupole magnets, and a baseline value is recorded

\footnotetext{
* Work supported by U.S. Department of Energy, Office of Basic Energy Sciences under Contract No. W-31-109-ENG-38.

† emery@aps.anl.gov
}

at the 0-A setting of the CPU main coil. The set points for the skew-quadrupole correcting magnet would then be adjusted using some algorithm at each main coil set point until the beam size is restored to its baseline value. The set points are then collected together to form the feedforward table.

During initial determination of these optimum set points, we found that we were not able to restore the baseline value of vertical beam size for all CPU set points. It was as though another variable source of skew quadrupole errors was present. We realized that two skew quadrupole correctors were necessary to compensate the skew quadrupole error of the CPU, because the center of mass of the distributed skew quadrupole field error was moving, and not necessarily staying at one end of the CPU.

The difficulty of using only one corrector for an error source located some distance away can be demonstrated through simulation of the correction of a trajectory in the storage ring generated by a general skew quadrupole error. The simulation presented here includes two skew quadrupole correctors at the ends of the CPU. The correction is analogous to the 3-magnet bump of orbit correction.

Fortunately, it was pointed out by I. Vasserman [1] that the relatively unimportant skew octupole magnet has a significant systematic skew quadrupole field, and it happens to be located at the opposite end of the CPU from the regular skew quadrupole magnet corrector. Vasserman suggested using this octupole magnet as the "other" skew quadrupole corrector.

An experimental 2D scan of the two skew correctors revealed the dependence of vertical beam size on their set points. An optimizer was used to determine their optimum set points for each CPU set point. Finally, the CPU was scanned in feedforward mode to verify the skew quadrupole correction.

\section{SIMULATION}

The general correction of a local skew quadrupole field error requires four independent skew quadrupole corrector magnets nearby. Because there are only drift spaces between the source and the corrections, two correctors suffice.

Figure 1 shows the layout of the $\mathrm{CPU}$ and the corrector coils in straight section ID4. The tracking code elegant [2] is used to generate a perturbed vertical dispersion. The vertical dispersion is used as a substitute for the more complicated and general $4 \times 4$ coupled transfer matrix. If we can compensate the vertical dispersion, then we can com- 
pensate the transfer matrix (as long as there are only drift spaces between skew elements). We calculate the vertical dispersion of the whole ring with a perturber centered in the ID4 CPU. Then we run the elegant optimizer under two conditions: using only one corrector downstream of the perturbation and using one at each end. Figure 2 shows the vertical dispersion compensation with only one variable, and it is seen to be insufficient in compensating the vertical dispersion everywhere in the ring. With two correctors the vertical dispersion is completely compensated outside the straight section (not shown).

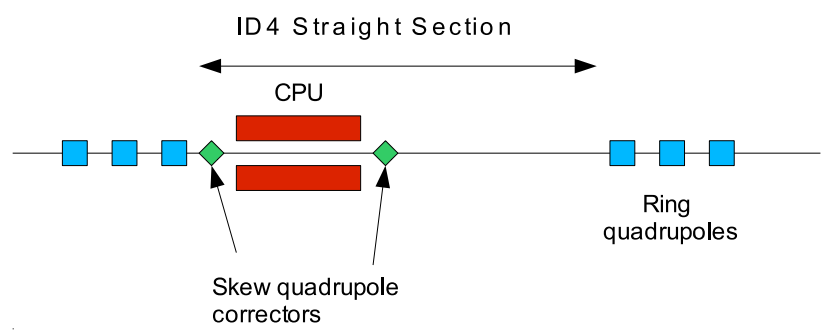

Figure 1: Layout of ID4 straight section showing the skew quadrupole correctors on either side of the CPU. One of the correctors is actually a skew octupole with large systematic skew quadrupole field.
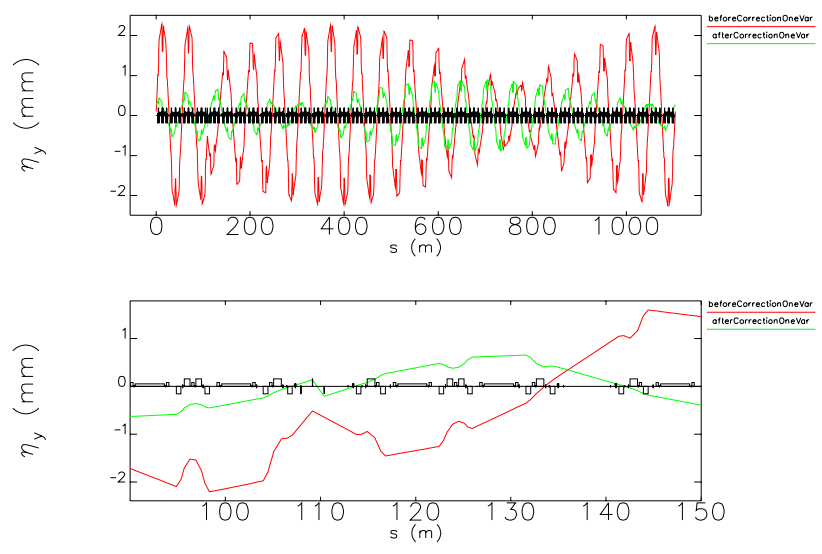

Figure 2: Vertical dispersion before and after a correction using one corrector, the original concept for coupling compensation. The perturber is located at the center of the CPU $(s=109.2 \mathrm{~m})$, the corrector is at the downstream end of the CPU $(s=110.4 \mathrm{~m})$. The correction is not sufficient to correct the global vertical dispersion.

\section{MEASUREMENTS}

We tested the idea of using the two skew correctors on either side of the real CPU for minimizing the vertical beam size. The correction of skew quadrupole errors for several CPU set points was successful. We also did a $2 \mathrm{D}$ scan of the skew quadrupole and octupole settings and measured the vertical beam size. The data plotted in the contour plot of Figure 3 reveals the simple nature of the vertical beam size dependence of the skew quadrupole correctors. These particular data were taken for a maximum CPU set point of $336 \mathrm{~A}$ in $\mathrm{CW}$ mode. The contour plot shows a valley for the vertical beam size with a relatively broad minimum. The minimum is well enough defined, relative to the noise of the measurement, that it is reached consistently with the EPICS optimizer sddsoptimize [3,4]. The optimum corrector set points vary smoothly with the CPU set point, and the changing ratio indicates that the effective skew quadrupole source center moves within the CPU.

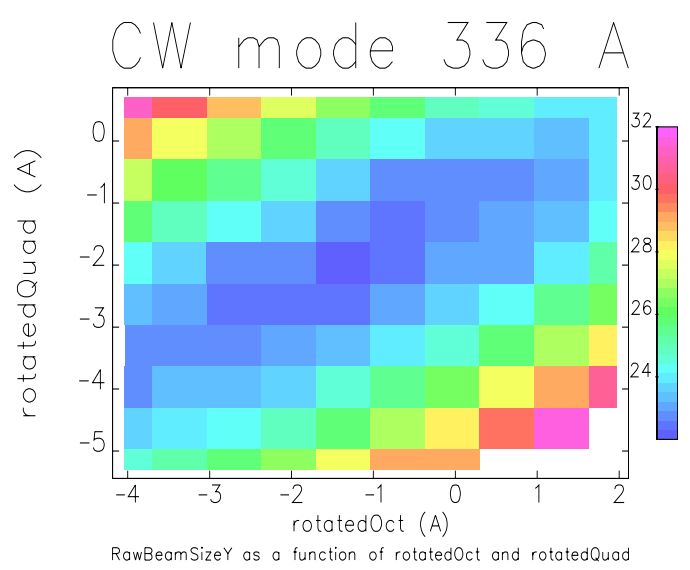

Figure 3: Contour plot of vertical beam size from a twodimensional scan of skew correctors for the CPU set point of $336 \mathrm{~A}$ in $\mathrm{CW}$ polarization mode. The solution obtained by the optimizer is -1.0 A for the skew octupole and -2.3 A for the skew quadrupole, which is at the minimum shown here.

\section{RESULTS}

A graphical user interface was written and run for correcting the $\mathrm{CW}$ and $\mathrm{CCW}$ modes of the $\mathrm{CPU}$. The linear polarization DC modes, $\mathrm{H}$ and $\mathrm{V}$, didn't require correction as the vertical beam size changed little during those CPU main coil scans. The determination of feedforward tables takes about one hour of study time for each mode. Figure 4 is a time plot of the vertical beam size when the CPU main coil is scanned with the original skew magnet set points. The change in vertical beam size appears small and not important. However, the resolution of the measurement optics of about $22 \mu \mathrm{m}$ has not been removed for the data. The beam size variation is actually significant.

Figure 5 shows the vertical beam size as a function of CPU main coil (same data as in Figure 4). Figure 6 shows the vertical beam size as a function of CPU main coil after correction. Note that the stability of the beam size is on the order of the measurement noise. Note also the change in the corrector set points curve from Figure 5.

Figure 7 shows the vertical beam size as a function of $\mathrm{CPU}$ main coil for the $\mathrm{CCW}$ mode (the other mode). Figure 8 shows the vertical beam size as a function of CPU main coil after correction. Again there is much improvement. 


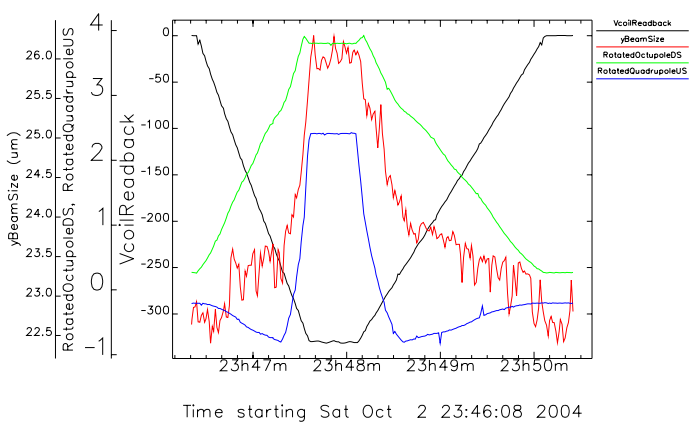

Figure 4: Up and down ramp of CPU main coil in $\mathrm{CW}$ mode showing the vertical beam size and the original set points of the skew correction coils.

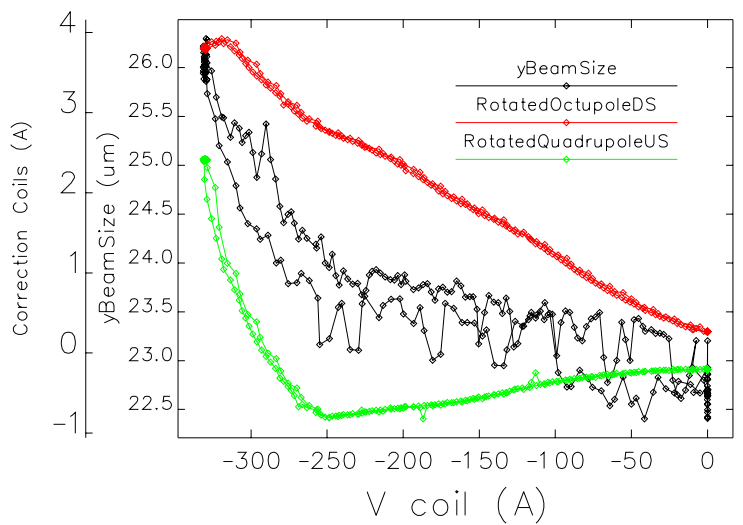

Figure 5: The vertical beam size and the original set points of the skew correction coils as a function of CPU main coil in CW mode. Both directions of the CPU ramp are shown. The apparent hysteresis may be an artifact of the 3-s averaging in the image processing.

\section{CONCLUSION}

We have determined that a pair of correctors is required for compensating the skew quadrupole error of the CPU. The compensation was relatively easy to implement. This suggests that other wiggler applications in low verticalemittance rings (light sources or damping rings) may require pairs of skew quadrupole correctors as well.

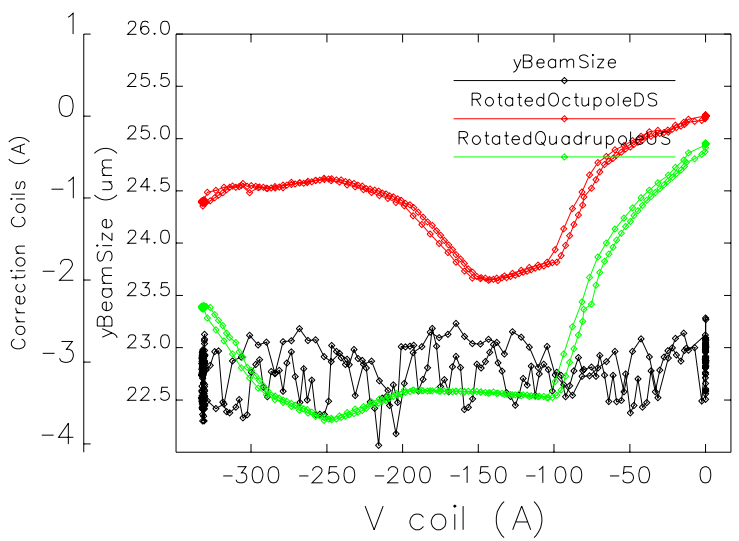

Figure 6: The vertical beam size and the optimized set points of the skew correction coils as a function of CPU main coil in $\mathrm{CW}$ mode. Both directions of the $\mathrm{CPU}$ ramp are shown.

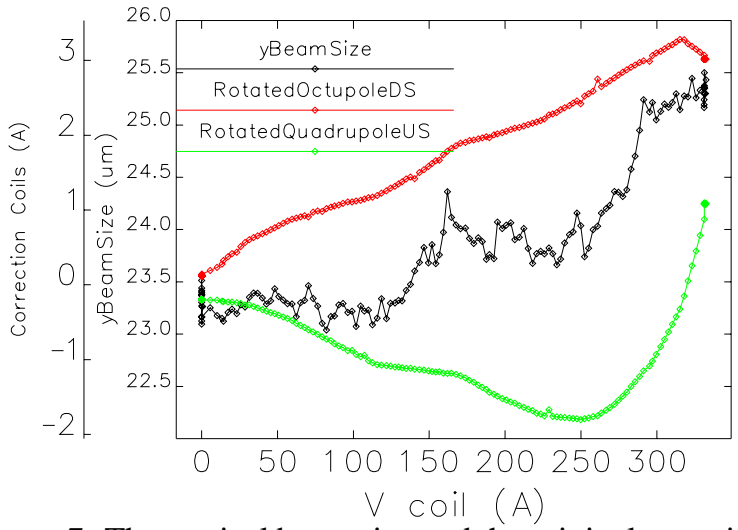

Figure 7: The vertical beam size and the original set points of the skew correction coils as a function of CPU main coil in $\mathrm{CCW}$ mode. Both directions of the $\mathrm{CPU}$ ramp are shown.

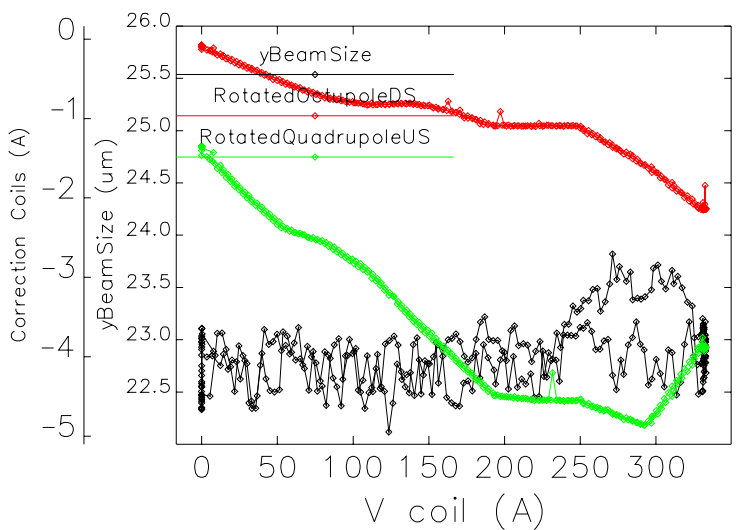

Figure 8: The vertical beam size and the optimized set points of the skew correction coils as a function of CPU main coil in $\mathrm{CCW}$ mode. Both directions of the CPU ramp are shown.

\section{ACKNOWLEDGEMENT}

I. Vasserman (XFD-MD) had the idea of using the octupole magnet as the second corrector after I asked him if a second skew quadrupole magnet could be installed near the CPU.

\section{REFERENCES}

[1] I. Vasserman, private communication.

[2] M. Borland, "elegant: A Flexible SDDS-Compliant Code for Accelerator Simulation," Advanced Photon Source Light Source Note LS-287, September 2000.

[3] L. Emery, M. Borland, H. Shang, "Use of a GeneralPurpose Optimization Module in Accelerator Control," Proceedings of the IEEE 2003 Particle Accelerator Conference, May 12-16, 2003, Portland, Oregon, pp. 2330-2332, http://www.jacow.org

[4] H. Shang, R. Soliday, L. Emery, M. Borland, "New Features in the SDDS-Compliant EPICS Toolkit," Proceedings of the IEEE 2003 Particle Accelerator Conference, May 12-16, 2003, Portland, Oregon, pp. 3470-3472, http://www.jacow.org 